\title{
A paradigm shift in mathematical physics, Part 4: Quantum computers and the local realism of all 4 Bell states
}

\author{
Jeffrey H. Boyd
}

Retired

57 Woods Road, Bethany, CT 06524, USA

\begin{abstract}
jeffreyhboyd@gmail.com

Can quantum information systems be understood using local realism? The consensus is "No." Quantum information is based on qubits and Bell states. According to conventional wisdom these cannot be understood using local realism. Invariably "local realism" is assumed, incorrectly, to refer only to the Einstein, Podolsky and Rosen (EPR) model. Today a radically different model of local realism has arisen. The Theory of Elementary Waves (TEW) is incompatible with Einstein's picture of "reality" but nevertheless is local and realistic. We show that the Bell test experiments that invalidate EPR, validate TEW! This article uses TEW to reproduce all four Bell states. We also show that TEW can explain an experiment using remote entangled photons that have no shared history, which are entangled because of entanglement swapping. The implications of our study for quantum information theory are unclear, except that the term "nonlocal" should be replaced with a more precise term. "Elementary ray" is the verifiable and precisely defined term that should replace it. This paradigm shift could inspire a new generation of quantum information experiments.
\end{abstract}

\section{Indexing terms/Keywords}

Theory of Elementary Waves, TEW, local realism, quantum computers, entanglement, bi-rays, EPR Bell states, entanglement swapping, quantum information

\section{Academic Discipline And Sub-Disciplines}

Physics, Quantum Physics, Foundations of quantum mechanics

\section{SUBJECT CLASSIFICATION}

Library of Congress Classification \#'s for Quantum Theory are from QC173.96 to QC174.52

for example: QC173.96 Quantum Mechanics Foundations or QC174.12.Q36 Quantum theory

\section{TYPE (METHOD/APPROACH)}

This is the fourth in a series of articles on a paradigm shift of tectonic proportions caused by the Theory of Elementary Waves (TEW). Whereas an ordinary elementary ray has the structure of a cylindrical helix, the elementary bi-rays discussed here have the structure of a double helix, the two strands of which are travelling in opposite directions at the speed of light. The word "entanglement" in TEW means that two particles are following the same bi-ray. Such a bi-ray induces a relationship between the two particles based on contingency variables. We show that this bi-ray math is able to reproduce the results of quantum math under conditions of the four Bell states. This math is applied to a bright source of entangled photons created at the University of Innsbruck by Kwiat, Mattle, Weinfurter, et. al.

\section{COUNCIL FOR INNOVATIVE RESEARCH}

Peer Review Research Publishing System

JOURNAL: JOURNAL OF ADVANCES IN MATHEMATICS

VOL.11, No.7

www.cirjam.com, editorjam@gmail.com 


\section{PART 1. Introduction}

Niels Bohr said that physical reality at the quantum scale either does not exist, or is structured in a manner so drastically different than the classical world we live in, that humans cannot comprehend it.[1] We should avoid talking about the quantum world, he said, and talk about that which we can see, which is the meter readings of our experiments. The Copenhagen school of quantum mathematics (QM) developed a science of meter readings, and made every effort to avoid speculating about what must exist down inside reality at the quantum scale to produce such meter readings. QM can be defined as the science of "observables," not the science of physical reality independent of the observer.

One of the themes of this article is that a new theory, the Theory of Elementary Waves (TEW) [2-11], has emerged as a complementary science: the science of physical reality independent of the observer. TEW is a realistic theory, radically different than Einstein's ideas. TEW is "local" even though John Bell would classify it as non-local. In the "local" versus "non-local" debate TEW is both, like field theories. It is a theory that is consistent with all Bell test experiments. This article will introduce for the first time a way to translate QM equations to TEW and vice-versa.

Bohr's portrayal of nature as unintelligible was reiterated in John Bell's picture of the quantum world.[12-14] From Bell, that anti-reality view infected all of quantum informational theory. But that which is unintelligible from one viewpoint, might be simple and orderly from a different viewpoint. If we adopt the viewpoint of TEW, the mysteries of nature are unlocked. What emerges is not Einstein's concept of nature, nor is it that of most lay people, but an unfamiliar world, different than anyone expected.

Quantum information is the study of information processing that can be accomplished using quantum mechanical systems.[15-18] If we adopt TEW as our framework, that has a subtle but pervasive influence on how we think about quantum computers and other quantum informational devices. Consider for example this sentence from an essay about quantum computers: "Bell states have the peculiar property that the particles always 'know' about each other even if they are separated by huge distances; this property is commonly associated with the 'non-locality' of quantum mechanics."[17] We claim this sentence is false. As we said in the Abstract, the term "non-locality" should be replaced by a more accurate and precise term: "elementary rays."

In this article we will focus on a 1995 article by Kwiat, Mattle, Weinfurter et. al.[19] which presents experimental data about a piece of optical equipment they built at the University of Innsbruck that produces entangled photons in a physical analog of all four of the Bell states. We will show how TEW can explain the data in the Kwiat article.

\subsection{History of the Bell test experiments}

The quantum information story starts with an article published in 1935 by Einstein, Podolsky and Rosen (EPR).[20] Every history of quantum information start with this article, but we interpret that history in a startling new way.

An equally famous rebuttal to EPR was published in 1964 by John Bell. The dispute was as follows: Einstein et. al. pictured a pair of spin $1 / 2$ particles which were formed in a singlet spin state, split apart, and are now moving in opposite directions. Quantum math (QM) tells us that if the spin of one $\sigma_{1}$ is found to be up, the spin of the other $\sigma_{2}$ must be down. More specifically, it tells us that the two spins are in PERFECT anti-parallel alignment. Bell wrote: "If measurement of the component $\sigma_{1} g a$, where $\mathbf{a}$ is some unit vector, yields a value of +1 then, according to quantum mechanics, measurement of $\stackrel{r}{\sigma_{2}}$ ga must yield a value of -1 and vice versa."

EPR and Bell focused on different aspects of this situation. Einstein et. al. emphasized that each particle has a specific spin BEFORE it is measured, and he said that QM cannot account for that. Therefore there must be something else, commonly called a "hidden variable," that needs to be added to QM to account for this intrinsic spin. The variable to be added would make QM "complete."

Bell on the other hand emphasized the word "PERFECT." He said that the QM picture is one of PERFECT anti-parallel alignment. Based on probability theory he showed that any "hidden variable" could never provide perfect agreement, only approximate agreement. If $\mathbf{a}$ is a unit vector used to measure $g_{1}$, and $b$ is the corresponding unit vector used to measure $\stackrel{I}{\sigma}_{2}$, then the expectation value of the product $\left\langle\sigma_{1}^{r} g \mathrm{ga} \stackrel{\sigma}{r}_{2} \mathrm{gb}\right\rangle$ defined by probability theory would need to equal to the expectation value of $\breve{G} \mathrm{gb}$ defined by QM. But Bell shows from probability theory this is impossible. His proof is elegant and compelling. A probabilistic "hidden variable" can never provide PERFECT anti-parallel alignment. Bell concludes that Einstein's metaphysics is wrong. The two particles do not have any spin before they are measured.
According to QM when you measure $\sigma_{1}$ ga for one particle, that triggers wavefunction collapse so that the pair of particles instantaneously acquire opposite spins. Prior to measurement neither particle has a specific spin - only a superposition, and when you measure the spin of one particle that measurement creates reality, and instantly (faster than light) a signal is sent to the other particle to instantly acquire the opposite spin.

One implication is that there is no such thing as reality independent of the observer. As David Mermin later said, "Science has proved that the moon only exists when people look at it!"[21-22] Another example is Schrödinger's cat, which is both dead and alive until we open the lid of the box and look. 


\section{ISSN 2347-1921}

Another implication is that QM is capable of sending a signal faster than the speed of light: instantaneous! Thus in the subsequent Bell test experiments there was a successful effort to show that the results occur faster than the speed of light.

The fact that in 1964 Bell defined these issues mathematically meant that it should be possible empirically to test whether Einstein or Bell was correct. This triggered three decades of experiments, called "Bell test experiments."

It proved difficult to wrestle with a pair of spin $1 / 2$ particles in the lab. The equipment is cumbersome. A pair of polarized photons was more manageable and practical. Clauser, Horne, Shimony and Holt (CHSH)[23] designed an experiment to test Einstein versus Bell using polarized photons, and in 1982 Alain Aspect's research team built exactly the experiment designed by $\mathrm{CHSH}$, and showed that Einstein was wrong.[24] A variable "S" can be defined, which we call the " $\mathrm{CHSH}$ variable", which would be bounded $|S| \leq 2$ if Einstein were correct, but would have a wider range $|S| \leq 2 \sqrt{2}$ if Bell were correct.[25] Thirty years of empirical research showed that the Einstein boundary $|S| \leq 2$ was always violated. Today all loopholes have been plugged up. Einstein was wrong.

Note that two different ideas got tangled together: a mathematical idea from John Bell and a metaphysical idea that was originally stated by Niels Bohr. Any mathematician can tell you that those are different disciplines. It leads to fuzzy thinking if they are muddled together. Philosophy and math simply don't mix. Philosophers drive mathematicians crazy, and vice versa. The mathematical and empirical proof that Bell was correct and Einstein wrong, does not mean that one metaphysics is correct and the other wrong. Perhaps there is a different definition of "local realism" that no one knew about back then.

Quantum physicists sometimes say that a particle on the other side of the galaxy can instantly affect a particle in our lab, without any method of doing so. That is "nonlocality." These ideas are embedded in the very fabric of quantum information theory. They are woven into the assumptions that quantum scientists take for granted.

We claim these assumptions are wrong. To say that Bell was correct and Einstein was wrong, only means that $|S| \leq 2$ was violated in Bell test experiments. The experiments were designed to drive a wedge between Einstein and Bell, and they successfully did so.

Today there is a third option. We now have TEW, which is a picture of everyday nature that is local and realistic, yet TEW is drastically different than anything Einstein or Bell ever imagined. We will demonstrate that TEW arrives at exactly the same results as QM in four Bell test experiments. For TEW, as for Bell, the range of the variable "S" is $|S| \leq 2 \sqrt{2}$.

A similar metaphysical debate raged in physics during the eighteenth and nineteenth century. Newton's theory of gravity was interpreted as meaning that planets were affected by gravity without anything intervening between the sun and the planet. Maxwell's equations introduced the idea of a field, thus resolving the "loval" versus "nonlocal" debate.

\subsection{The emergence of TEW}

No one has ever attempted to connect quantum computers and TEW. We plan to integrate these divergent fields into a unity. To get there we need to discuss not only quantum information theory, but also TEW, which we will now do. To understand TEW we need to start with an error in the foundations of QM.

The root of all evil in quantum mathematics (QM) is a covert assumption made by Bohr, Einstein, Heisenberg, Schrödinger, Pauli, Bohm, de Broglie, Born, Ehrenfest, Dirac, Feynman and ALL the founding fathers. They assumed that waves and particles travel in the same direction. This seemed so obvious that they were not aware they were making an assumption. They never mentioned it in their writings, never thought about it, never sought any empirical evidence to support that assumption. To reiterate: QM is built on an unstated and untested assumption.

Too bad! Had they sought evidence they would have found none. We now know that the preponderance of evidence points in the opposite direction. We showed in earlier publications that wave particle duality experiments fail to prove that waves and particles travel in the same direction duality.

Because of this original assumption the founding fathers were unable to picture how nature works at the quantum level. Therefore they were forced to build an abstract theory unrelated to physical reality except for "observables" dropping out of the machinery from time to time. Many quantum experts followed Bohr in declaring there is no such thing as physical nature at the quantum level, or that it is unintelligible. The usual approach is to avoid thinking about it, and declare, "Shut up and do the math!"

The incredible power of mathematics is evident in QM. Even though the physicists did not understand the quantum world, their mathematics was led by empirical studies and was therefore shaped so that the equations grasp nature even though the physicists had heads full of nonsense. Who needs a brain? All you need is mathematics!

If you assume that waves travel in the same direction as particles, you end up with wavefunction collapse located at the detector, which is wrong. There is zero hope for you understanding how nature works, if you think collapse is located at the detector.

If particles and waves travel in opposite directions then probability amplitudes from the detectors can travel to the particle source before an experiment is conducted. Although the elementary rays of TEW move at the speed of light, they can be established as a network connecting Alice and Bob's equipment long before the scientists come to work on Monday 
morning, long before the electricity is turned on. Therefore their influence appears to be "faster than the speed of light" when an experiment is conducted.

\section{PART 2. The mathematics of bi-rays}

\subsection{Advanced TEW and bi-rays}

TEW consists of two bodies of mathematics, which can be called "introductory," and "advanced TEW." They do not give the same results when applied to a problem. For example, if you were to analyze a double slit experiment using the model of a photon following a bi-ray, the results would be wrong. So far the only arena in which we know that advanced TEW math applies is in experiments involving entangled particles.

"Entanglement" is a core concept in quantum computers. But the term has a different definition in QM than in TEW. In QM it means that two or more particles cannot be understood separately because they are part of the same wave equation. In TEW "entanglement" means that two or more particles are following the same bi-ray.

\subsection{What does a bi-ray look like?}

An elementary ray amplitude travels in the opposite direction of, and precedes, the particle. Particles follow them backwards. Earlier in this series we showed that these rays have the form of a cylindrical helix traveling at the speed of light down the long axis of the corkscrew. We use the symbol $\boldsymbol{E}$ to refer to an elementary ray. A bi-ray is defined a pair of coaxial elementary rays traveling in opposite directions. An entangled pair of photons follow such an object.

We continue the convention of using the symbol $\mathscr{E}_{\mathrm{R}}$ to denote an elementary mono-ray moving to the right, and $\mathbb{E}_{\mathrm{L}}$ to denote an elementary mono-ray moving to the left. A bi-ray is symbolized by: ${ }_{R}{ }_{R}\left(\hat{u}_{\hat{R}} \mathbb{L}\right.$. We use red and blue colors to simplify reading.

A quantum amplitude is the mathematical way of referring to the elementary ray amplitude, which is a complex number: $\mathrm{A}=|\mathrm{A}| e^{i \phi}$. It is important to remember that the $\mathrm{QM}$ equivalent is the elementary ray amplitude, not the elementary ray as a whole. According to that logic a bi-ray amplitude should be the amplitudes of a pair of coaxial elementary rays:

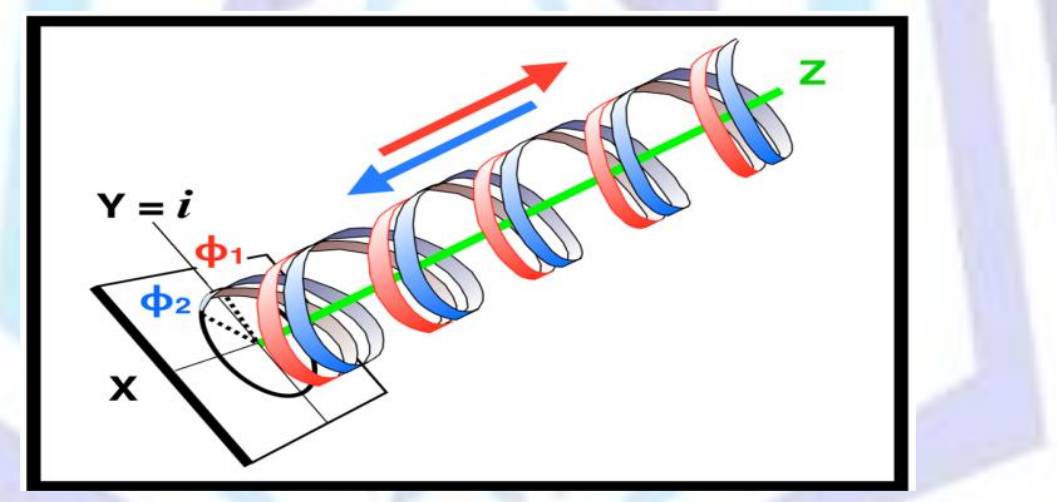

Fig. 1 Our model of a bi-ray $\mathbb{R}_{R}\left(\mathbb{u} \hat{u}_{L}\right.$ shows two helices moving in opposite directions.

In Figure 1 both cylindrical helices have the same radius, which is to say $\left|A_{R}\right| \hat{\mathrm{E}}\left|A_{L}\right| \equiv \mathrm{A}$. Although we portray the helices in Figure 1 as if they had a non-zero radius in Cartesian space, that is not true. The Cartesian diameter is zero. An elementary ray occupies the boundary between Cartesian and Hilbert space. The Hilbert space properties are the radius of the helix, and the angle of rotation (Figure 1). The Cartesian properties include a location in Cartesian space and a velocity, namely the speed of light. The best way to think of the amplitude is as a cross-section of the bi-ray, at which point we find that each helix has a complex amplitude, as noted above.

Each part of the bi-ray conveys zero energy:

$$
E\left(\mathbb{R}_{R}\right)=0 \quad \text { and } \quad E\left(\left(_{L}\right)=0\right. \text {. }
$$

The formula for the bi-ray helices are:

$$
\begin{gathered}
=\sin \left(\theta_{1}+\theta_{2}\right) \times \sin \left(\theta_{1}+\theta_{2}\right) \\
=\sin ^{2}\left(\theta_{1}+\theta_{2}\right)
\end{gathered}
$$

When discussing the Kwiat 1995 article below our primary focus is on the following equation:

$\psi_{\mathrm{E}} \equiv \Pi+\left(\mathbb{R}_{\mathrm{R}} \circledast \hat{\mathrm{u}} \mathrm{R}_{\mathrm{L}}\right)+\Pi$ 
where $\psi_{\mathrm{E}}$ denotes the entangled state of the system (the wavefunction) and

$\Pi$ denotes a particle: in this case a photon (see Figure 2). The probability of a photon $\Pi$ following a bi-ray is the amplitude of it following the red prong of the bi-ray times the amplitude of it following the blue prong.

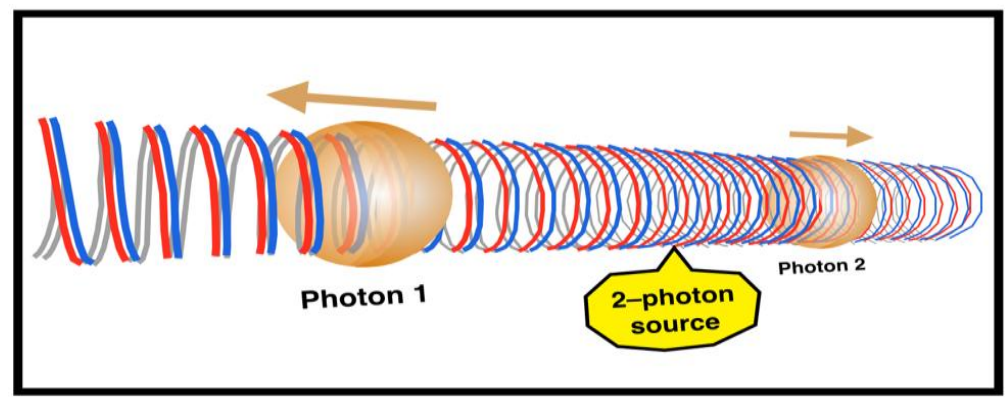

Fig 2. This is a diagram of $\Pi+\left(\mathbb{R}_{R}\left(\mathbb{u} \hat{\mathbb{R}}_{L}\right)+\Pi\right.$ with 2 photons moving away from a common source.

Although we have been discussing the angles $\phi_{1}$ and $\phi_{2}$, in a working model of a bi-ray it is the velocity of rotation (angular momentum) that is more interesting. This comes into focus when we discuss the frequency:

$$
v_{1}=\frac{\phi_{1}}{2 \pi t}=\frac{\omega_{1}}{2 \pi} \quad \text { and } \quad v_{2}=\frac{\phi_{2}}{2 \pi t}=\frac{\omega_{2}}{2 \pi} .
$$

Of particular interest is the wavelength of each of the mono-rays.

$$
\lambda_{1}=\frac{c}{v_{1}} \quad \text { and } \quad \lambda_{2}=\frac{c}{v_{2}}
$$

In Figures 1 and 2 the countervailing rays have the same wavelength. In the Kwiat article discussed below that is $\lambda_{{ }}=$ $\lambda_{B_{L}}=702.2 \mathrm{~nm}$. In other Bell test experiments, such as that by Aspect et al, the countervailing fays have wavelengths that are slightly different than each other.

We have a drastically different picture of "reality" than almost anyone else. According to our picture there is no waveparticle duality. Waves (also called "rays" or "quantum amplitudes") are not necessarily attached to particles. In any volume of space there are an infinite number of elementary rays and bi-rays, but only a finite number of particles. As Feynman showed in his book QED, particles follow quantum amplitudes, which convey no energy.[26] Our picture of reality differs from Feynman's only in that he assumed they both travel in the same direction, whereas we say they usually travel in opposite directions. At any point in space there are elementary rays of all frequencies, traveling in all directions at the speed of light. Particles are incapable of existing or moving without being attached to some elementary ray of the same frequency. The particles alone carry all the energy, momentum, and everything needed to make a detector "click." Elementary rays are invisible. The only way to know about them is by inference based on our study of the behavior of particles when they make our detectors "click."

\section{PART 3. Definition of the four Bell states}

Since this article focuses on the four Bell states, we will now define them. Each of two qubits can take values $|0\rangle$ or $|1\rangle$, or a linear combination of them: $(\alpha|0\rangle+\beta|1\rangle)$ or $(\alpha|0\rangle-\beta|1\rangle)$. We can define $\alpha=\beta=\frac{1}{\sqrt{2}}$. This leads to two definitions:

$$
\begin{aligned}
& |+\rangle \equiv \frac{1}{\sqrt{2}}(|0\rangle+|1\rangle) \quad \text { and } \\
& |-\rangle \equiv \frac{1}{\sqrt{2}}(|0\rangle-|1\rangle)
\end{aligned}
$$

We can take this $|+\rangle,|-\rangle$ as the basis for defining the anti-parallel correlation of Alice and Bob's data.[ 27] Next we define an entangled state of the system

$$
\left|\psi^{+}\right\rangle \equiv \frac{1}{\sqrt{2}}\left[\left(|+\rangle_{A} \otimes|-\rangle_{B}\right)+\left(|-\rangle_{A} \otimes|+\rangle_{B}\right)\right]
$$


which we will call "the first Bell state." In this state Alice and Bob's data are in perfect anti-parallel correlation, meaning that Alice's $|+\rangle_{A}$ corresponds to Bob's $|-\rangle_{B}$, and vice-versa.

Kwiat et. al. define a physical analog of equation 6 as follows:

$$
\left|\psi^{+}\right\rangle=\frac{1}{\sqrt{2}}\left(\left|H_{A}, \mathrm{~V}_{B}\right\rangle+\left|V_{A}, \mathrm{H}_{B}\right\rangle\right)
$$

There are four Bell states, which are maximally entangled states of a two cubit Hilbert space:

$$
\begin{aligned}
& \left|\psi^{+}\right\rangle \equiv \frac{1}{\sqrt{2}}\left[\left(|+\rangle_{A} \otimes|-\rangle_{B}\right)+\left(|-\rangle_{A} \otimes|+\rangle_{B}\right)\right] \\
& \left|\psi^{-}\right\rangle \equiv \frac{1}{\sqrt{2}}\left[\left(|+\rangle_{A} \otimes|-\rangle_{B}\right)-\left(|-\rangle_{A} \otimes|+\rangle_{B}\right)\right] \\
& \left|\xi^{+}\right\rangle \equiv \frac{1}{\sqrt{2}}\left[\left(|+\rangle_{A} \otimes|+\rangle_{B}\right)+\left(|-\rangle_{A} \otimes|-\rangle_{B}\right)\right] \\
& \left|\xi^{-}\right\rangle \equiv \frac{1}{\sqrt{2}}\left[\left(|+\rangle_{A} \otimes|+\rangle_{B}\right)-\left(|-\rangle_{A} \otimes|-\rangle_{B}\right)\right]
\end{aligned}
$$

These four states can serve as a basis in 2-cubit Hilbert space. Moving from math into the design of our optical hardware, these four Bell states have the following physical analogs in the Kwiat article:

$$
\begin{aligned}
& \left|\psi^{+}\right\rangle=\frac{1}{\sqrt{2}}\left(\left|H_{1}, V_{2}\right\rangle+\left|V_{1}, H_{2}\right\rangle\right) \\
& \left|\psi^{-}\right\rangle=\frac{1}{\sqrt{2}}\left(\left|H_{1}, V_{2}\right\rangle-\left|V_{1}, H_{2}\right\rangle\right) \\
& \left|\xi^{+}\right\rangle=\frac{1}{\sqrt{2}}\left(\left|H_{1}, H_{2}\right\rangle+\left|V_{1}, V_{2}\right\rangle\right) \\
& \left|\xi^{-}\right\rangle=\frac{1}{\sqrt{2}}\left(\left|H_{1}, H_{2}\right\rangle-\left|V_{1}, V_{2}\right\rangle\right)
\end{aligned}
$$

\section{PART 4. A source of entangled photons}

These four Bell states are produced by the equipment we are about to discuss. As shown in Figure 11 (later in this article) these states are so drastically different from one another that even a noisy channel should be able to distinguish one state from another.

An essential component of many quantum information devices is to have a bright source of entangled photons. We will discuss in detail such a light source designed by a team at the University of Innsbruck led by Kwiat.[19] Other sources of entangled photons are available today [23-24], but we will limit our discussion to the device by Kwiat, et.al.

\subsection{A device built at University of Innsbruck by Kwiat's team}

In the article by Kwiat, Mattle, Weinfurter, et al. (1995), an argon ion laser of $150 \mathrm{~mW}$ pumps $351.1 \mathrm{~nm}$ photons into a beta barium borate (BBO) crystal where each photon is split by type II spontaneous parametric down-conversion into ordinary and extraordinary photons of $702.2 \mathrm{~nm}$ that are orthogonally polarized, and that leave the crystal along two overlapping cones, and are entangled where the cones intersect (see Figure 3).

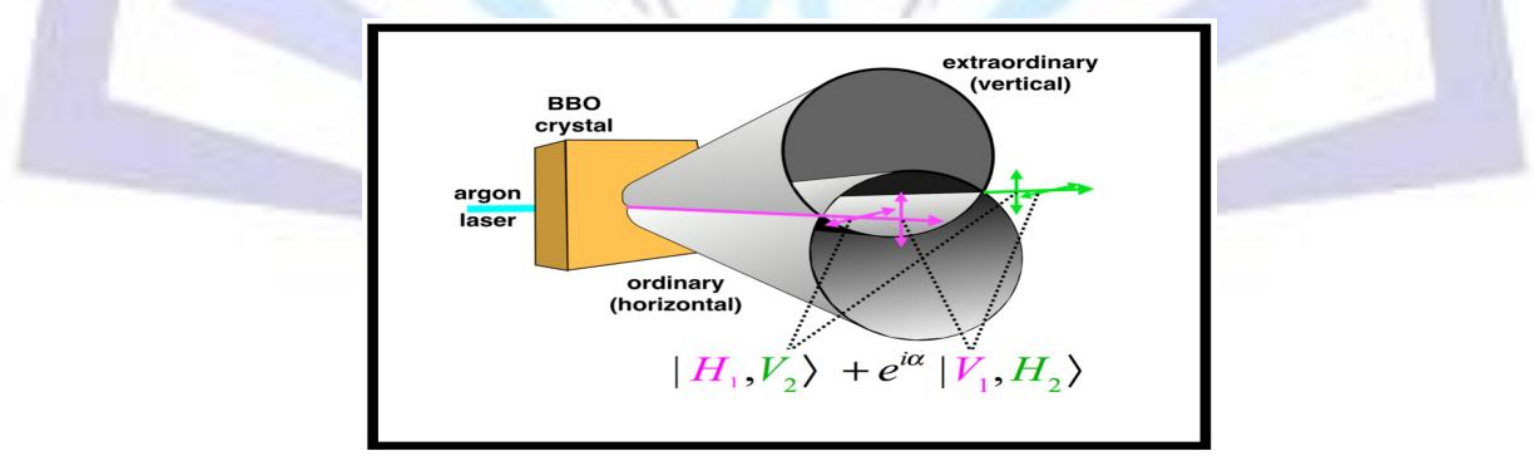

Fig. 3. At the intersection of the two cones entangled photons are produced, orthogonal to one another.

When we say the photons are "entangled," what we mean in TEW is that both photons are following the same bi-ray, as we said earlier.

Figure 3 demonstrates that photons in the entangled state are described by this formula:

$$
|\psi\rangle=\frac{1}{\sqrt{2}}\left(\left|H_{1}, V_{2}\right\rangle+e^{i \alpha}\left|V_{1}, H_{2}\right\rangle\right)
$$

The relative phase $\alpha$ comes from the crystal birefringence, which is adjusted by the researchers so $\alpha$ takes values of zero or $\pi$. By rotating the quarter and half wave plates (not shown in Figure 3 ) they produce any one of the four EPR-Bell states: see equations 7 and $10-12$ above. 
These four Bell states constitute a complete, maximally entangled basis for a two-particle Hilbert space. Table 1 summarizes information found in the Kwiat article.

\section{Table 1}

\section{Information from the Kwiat article}

$$
\begin{aligned}
& \text { Bell state \# Abbreviation Coincidence Rate } \\
& \text { 1. }\left|\psi^{+}\right\rangle=\frac{1}{\sqrt{2}}\left(\left|H_{1}, V_{2}\right\rangle+\left|V_{1}, H_{2}\right\rangle\right) \ldots(\mathrm{HV}+\mathrm{VH}) \ldots \mathrm{C}=\sin ^{2}\left(\theta_{1}+\theta_{2}\right) \\
& \text { 2. }\left|\psi^{-}\right\rangle=\frac{1}{\sqrt{2}}\left(\left|H_{1}, V_{2}\right\rangle-\left|V_{1}, H_{2}\right\rangle\right) \ldots(\mathrm{HV}-\mathrm{VH}) \ldots \mathrm{C}=\sin ^{2}\left(\theta_{1}-\theta_{2}\right) \\
& \text { 3. }\left|\xi^{+}\right\rangle=\frac{1}{\sqrt{2}}\left(\left|H_{1}, H_{2}\right\rangle+\left|V_{1}, V_{2}\right\rangle\right) \ldots(\mathrm{HH}+\mathrm{VV}) \ldots \mathrm{C}=\cos ^{2}\left(\boldsymbol{\theta}_{1}-\boldsymbol{\theta}_{2}\right) \\
& \text { 4. }\left|\xi^{-}\right\rangle=\frac{1}{\sqrt{2}}\left(\left|H_{1}, H_{2}\right\rangle-\left|V_{1}, V_{2}\right\rangle\right) \ldots(\mathrm{HH}-\mathrm{VV}) \ldots \mathrm{C}=\cos ^{2}\left(\boldsymbol{\theta}_{1}+\boldsymbol{\theta}_{2}\right)
\end{aligned}
$$

Table 1 provides a synopsis of information available in the Kwiat article.

\section{PART 5. Bi-ray explanation of the 4 Bell states}

The TEW thesis is that these photons are following elementary rays, one prong of which is traveling in the opposite direction. The primary determinant of our trigonometry will be that the red elementary ray of polarization HV exists on both sides of the source. However these rays are twisted as they pass through the source. The $\mathrm{H}_{1}$ on the left corresponds to the $V_{2}$ in the right, and the $V_{1}$ on the left corresponds to the $\mathrm{H}_{2}$ in the right ray. This corresponds to how the two photons are polarized.

\subsection{The first Bell state: $\left|\psi^{+}\right\rangle=\frac{1}{\sqrt{2}}\left(\left|H_{1}, V_{2}\right\rangle+\left|V_{1}, H_{2}\right\rangle\right)$ \\ (equation 7)}

When we diagram this, we represent the twist inside the source by an "S" curve. Figure 4 shows the bi-ray, with the blue mono-rays on the bottom doing precisely what the red rays did, in reverse direction.

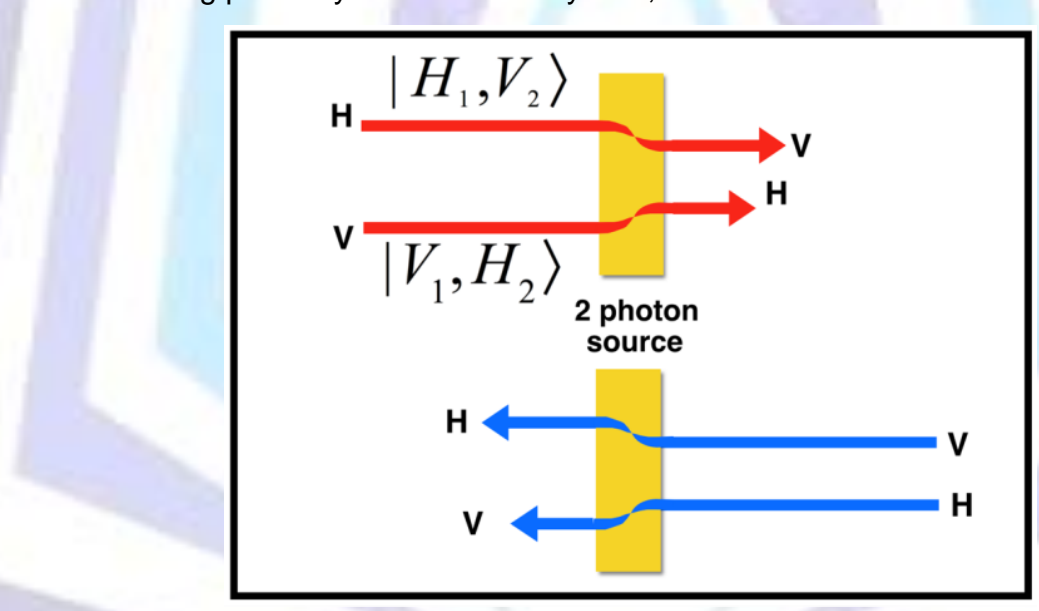

Fig. 4 Showing how elementary rays, crossing the photon source, twist by $\pm \pi / 2$ inside that source.

Focusing on the top red ribbon in Figure 4, we have interpreted that when the top horizontal vector $\mathrm{H}_{1}$ on the left passes through the source (yellow rectangle) it rotates by $-\pi / 2$ to become a vertical vector $V_{2}$ on the right. The angle $\pi / 2$ is in polar coordinates according to which a vertical vector has polar coordinate zero (angle between the $Z$ axis and the vertical vector). A horizontal vector makes an angle of $+\pi / 2$ relative to the $Z$ axis.

Similarly the second red ribbon in Figure 4 shows a vertical vector $V_{1}$ on the left rotating by $+\pi / 2$ to become the red horizontal $\mathrm{H}_{2}$ vector on the right. The blue ribbons are less interesting because they simply do the opposite of what the red ribbons have already done.

Although mono-rays have two eigenstates $(\mathrm{H}$ and $\mathrm{V})$ bi-rays have four, as follows: 


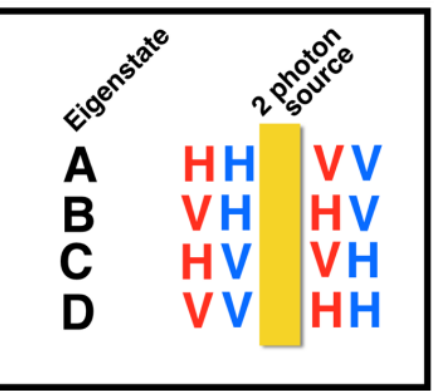

Fig. 5 This anagram shows the four eigenstates of the bi-rays.

Exactly the same information can be presented in another format: using clocks with one hand to symbolize the direction of polarization of each component, as shown in Figure 6.

\section{$\forall \mid ! C G$}

BOp

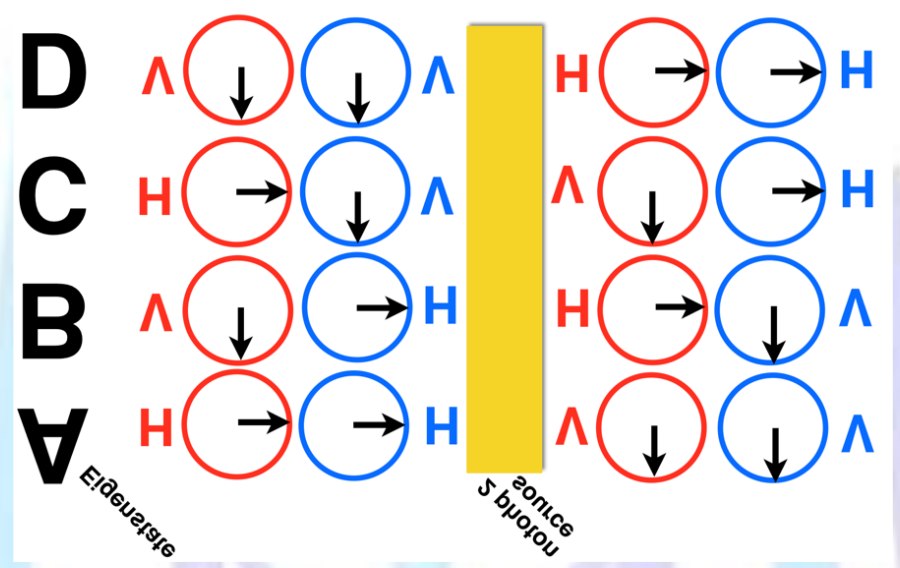

Fig. 6 These clock faces show the same information as was contained in the preceding anagram.

In the Bell test experiments Alice sets her polarizer at angle $\theta_{1}$, and records whether or not she sees a photon. Bob independently sets his polarizer angle at $\theta_{2}$, and records whether he simultaneously sees the other photon. Using green to represent Alice's polarizer angle, and purple for Bob's, we add that information to Figure 6, and thereby produce Figure 7.

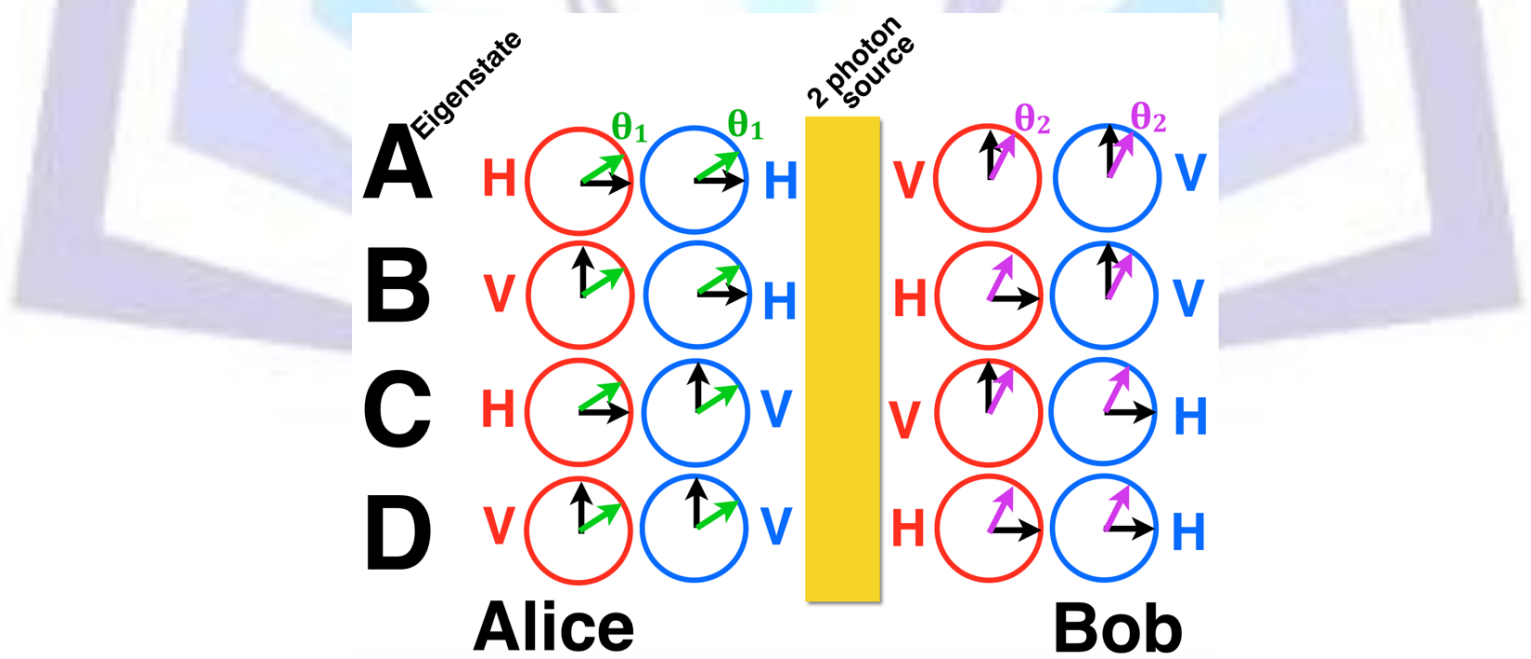

Fig. 7 We have added Alice's polarizer angle $\theta_{1}$ and Bob's angle $\theta_{2}$ to the preceding diagram.

This prepares us to read trigonometry directly from the clock faces. These are all unit vectors. Our trigonometry will be based on the magnitude of the projection of each polarizer angle onto the corresponding eigenstate.

The probability of Alice seeing a photon at polarizer angle $\theta_{1}$ and Bob simultaneously seeing the other photon at angle $\theta_{2}$ is (starting at the left side of the top row of Figure 6): 
Eigenstate

$$
\begin{array}{lll}
\mathrm{A} & +\cos \theta_{1} \cos \theta_{1} & \sin \theta_{2} \sin \theta_{2} \\
\mathrm{~B} & +\sin \theta_{1} \cos \theta_{1} & \cos \theta_{2} \sin \theta_{2} \\
\mathrm{C} & +\cos \theta_{1} \sin \theta_{1} & \sin \theta_{2} \cos \theta_{2} \\
\mathrm{D} & +\sin \theta_{1} \sin \theta_{1} & \cos \theta_{2} \cos \theta_{2}
\end{array}
$$

which can be factored:

$$
\begin{aligned}
= & \left(\cos \theta_{1} \sin \theta_{2}+\sin \theta_{1} \cos \theta_{2}\right) X \\
& \left(\cos \theta_{1} \sin \theta_{2}+\sin \theta_{1} \cos \theta_{2}\right)
\end{aligned}
$$

and simplified:

$$
\begin{gathered}
=\sin \left(\theta_{1}+\theta_{2}\right) X \sin \left(\theta_{1}+\theta_{2}\right) \\
=\sin ^{2}\left(\theta_{1}+\theta_{2}\right)
\end{gathered}
$$

Given the rule that the probability of a photon following a bi-ray is the amplitude of one ray times the amplitude of the other, our trig shows that the probability of Alice seeing a photon at polarizer angle $\theta_{1}$ and Bob simultaneously seeing the other photon at angle $\theta_{2}$ is $\sin ^{2}\left(\theta_{1}+\theta_{2}\right)$. Table 1 of the Kwiat article (page 5339) says that is precisely what QM predicts and what their experiment found.

Let us review where we are, to avoid getting disoriented. Our goal is to show how TEW can account for all four of the Bell states in the Kwiat article. So far we have accomplished that for the first state. We took the information embedded in the kets of equation 7 , and used that as a visual roadmap for what the elementary rays are doing. Since these are bi-rays, we showed the ray crossing the photon source, twisting by $\pm \pi / 2$ as it does so. We turn now to second of the four Bell states.

\subsection{Second Bell state $\left|\psi^{-}\right\rangle=\frac{1}{\sqrt{2}}\left(\left|H_{1}, V_{2}\right\rangle-\left|V_{1}, H_{2}\right\rangle\right)$}

(equation 10)

We will now streamline and simplify our diagrams so as to contain only the essential information. From what is written above the reader can easily reconstruct how the trigonometry arises from the following diagram. In the diagram below the elementary rays twist by $\pm \pi / 2$ as they pass through the source.

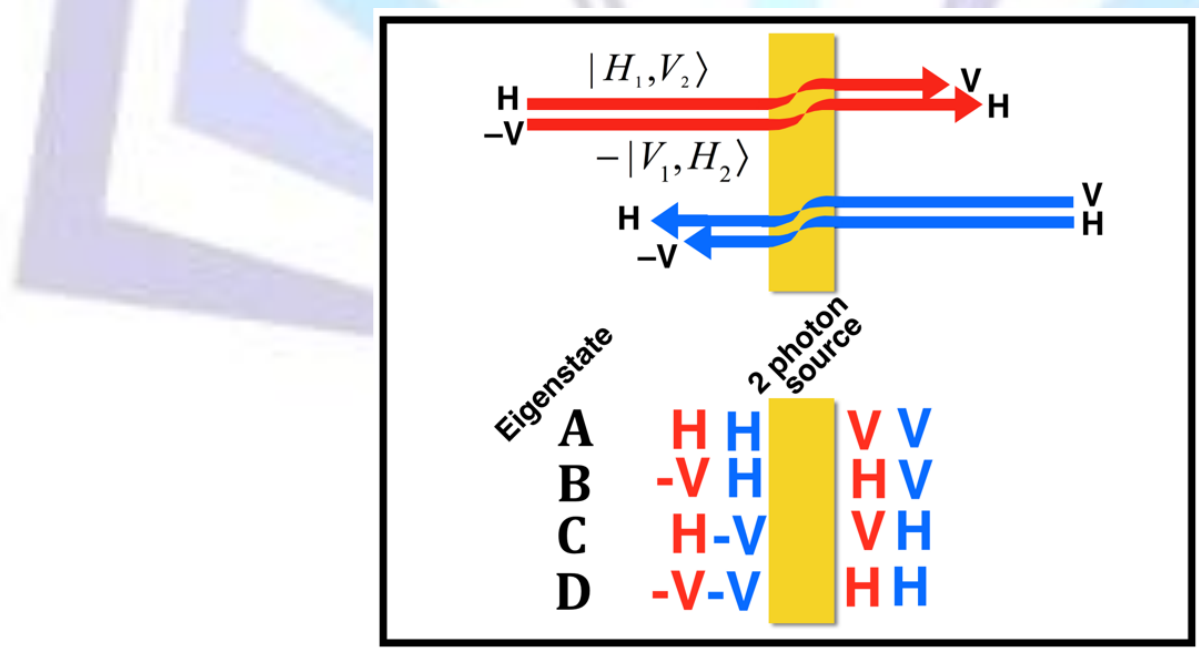

Fig. 8 Elementary rays (top) twist by $\pm \pi / 2$ in source; anagram summarizing that (bottom)

The probability of Alice seeing a photon at polarizer angle $\theta_{1}$ and Bob simultaneously seeing the other photon at angle $\theta_{2}$ is: 


\section{Eigenstate}

$$
\begin{array}{ccc}
\text { A } & +\cos \theta_{1} \cos \theta_{1} & \sin \theta_{2} \sin \theta_{2} \\
\mathrm{~B} & +\left(-\sin \theta_{1}\right) \cos \theta_{1} & \cos \theta_{2} \sin \theta_{2} \\
\mathrm{C} & +\cos \theta_{1}\left(-\sin \theta_{1}\right) & \sin \theta_{2} \cos \theta_{2} \\
\mathrm{D} & +\left(-\sin \theta_{1}\right)\left(-\sin \theta_{1}\right) & \cos \theta_{2} \cos \theta_{2} \\
= & +\cos \theta_{1} \cos \theta_{1} & \sin \theta_{2} \sin \theta_{2} \\
-\sin \theta_{1} \cos \theta_{1} & \cos \theta_{2} \sin \theta_{2} \\
\breve{G}_{\mathrm{G}} \theta_{1} \sin \theta_{1} & \sin \theta_{2} \cos \theta_{2} \\
+\sin \theta_{1} \sin \theta_{1} & \cos \theta_{2} \cos \theta_{2}
\end{array}
$$

which can be factored:

$$
\begin{aligned}
= & \left(\cos \theta_{1} \sin \theta_{2} \breve{\mathrm{G}} \sin \theta_{1} \cos \theta_{2}\right) X \\
& \left(\cos \theta_{1} \sin \theta_{2} \breve{\mathrm{G}} \sin \theta_{1} \cos \theta_{2}\right)
\end{aligned}
$$

and simplified:

$$
\begin{gathered}
=\sin \left(\theta_{1} \breve{\mathrm{G}} \theta_{2}\right) \times \sin \left(\theta_{1} \breve{\mathrm{G}} \theta_{2}\right) \\
=\sin ^{2}\left(\theta_{1} \breve{\mathrm{G}} \theta_{2}\right)
\end{gathered}
$$

Our trigonometry shows that the probability of Alice seeing a photon at polarizer angle $\theta_{1}$ and Bob simultaneously seeing the other photon at angle $\theta_{2}$ is $\sin ^{2}\left(\theta_{1} \breve{\mathrm{G}} \theta_{2}\right)$. Table 1 of the Kwiat article (page 5339) says that is precisely what QM predicts and what their experiment found.

5.3 Third Bell state $\left|\xi^{+}\right\rangle=\frac{1}{\sqrt{2}}\left(\left|H_{1}, H_{2}\right\rangle+\left|V_{1}, V_{2}\right\rangle\right)$ (equation 11)

In the next diagram the elementary rays pass straight through the photon source, without any rotation.

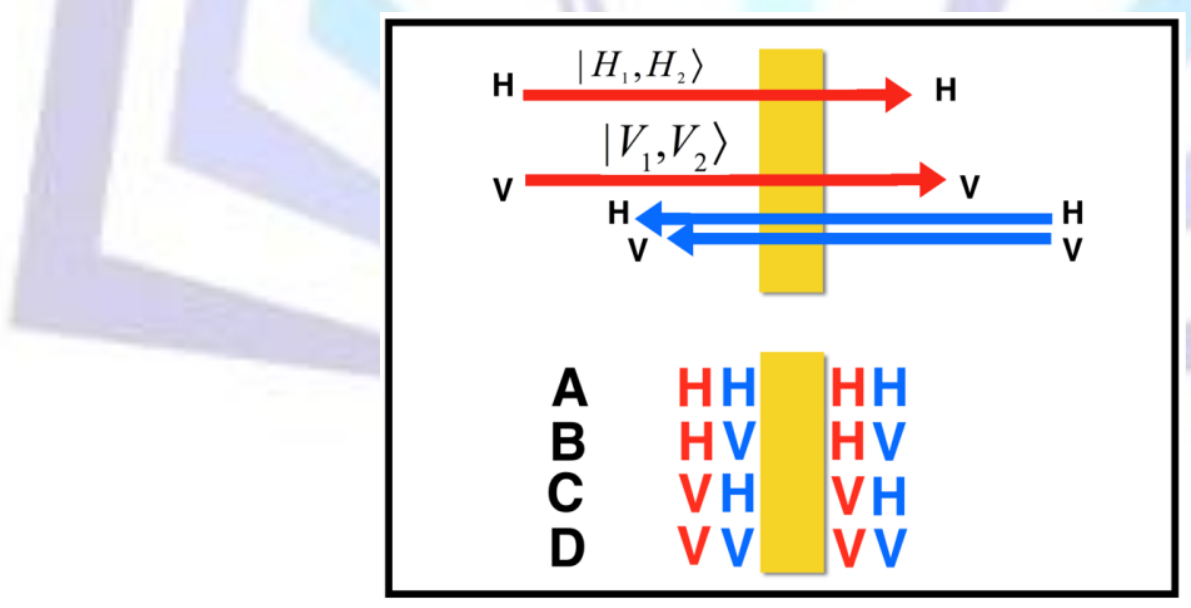

Fig. 9 Elementary rays (top) travel straight through the source; anagram summarizing that (bottom)

The probability of Alice seeing a photon at polarizer angle $\theta_{1}$ and Bob simultaneously seeing the other photon at angle $\theta_{2}$ is: 


\section{Eigenstate}

$$
\begin{array}{lll}
\text { A } & +\cos \theta_{1} \cos \theta_{1} & \cos \theta_{2} \cos \theta_{2} \\
\text { B } & +\cos \theta_{1} \sin \theta_{1} & \cos \theta_{2} \sin \theta_{2} \\
\text { C } & +\sin \theta_{1} \cos \theta_{1} & \sin \theta_{2} \cos \theta_{2} \\
\text { D } & +\sin \theta_{1} \sin \theta_{1} & \sin \theta_{2} \sin \theta_{2}
\end{array}
$$

which can be factored:

$$
\begin{aligned}
= & \left(\cos \theta_{1} \cos \theta_{2}+\sin \theta_{1} \sin \theta_{2}\right) X \\
& \left(\cos \theta_{1} \cos \theta_{2}+\sin \theta_{1} \sin \theta_{2}\right)
\end{aligned}
$$

and simplified:

$$
\begin{gathered}
=\cos \left(\theta_{1} \breve{\mathrm{G}} \theta_{2}\right) \times \cos \left(\theta_{1} \breve{\mathrm{G}} \theta_{2}\right) \\
=\cos ^{2}\left(\theta_{1} \breve{\mathrm{G}} \theta_{2}\right)
\end{gathered}
$$

Our trig shows that the probability of Alice seeing a photon at polarizer angle $\theta_{1}$ and Bob simultaneously seeing the other photon at angle $\theta_{2}$ is $\cos ^{2}\left(\theta_{1} \breve{\mathrm{G}} \theta_{2}\right)$. Table 1 of the Kwiat article (page 5339) says that is precisely what QM predicts and what their experiment found.

\subsection{Fourth Bell state $\left|\xi^{-}\right\rangle=\frac{1}{\sqrt{2}}\left(\left|H_{1}, H_{2}\right\rangle-\left|V_{1}, V_{2}\right\rangle\right) \quad$ (equation 12)}

In the next diagram one elementary rays passes straight through the photon source, but the other one rotates by $\pm \pi$ as it passes through the source.

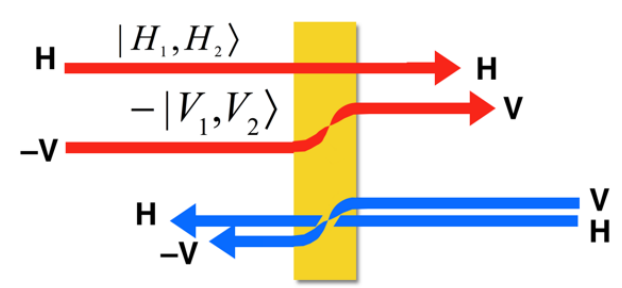
A $\mathrm{H} \mathrm{H} \quad \mathrm{HH}$
B $\mathrm{H}-\mathrm{V} \quad \mathrm{H} \mathrm{V}$
C - VH VH
D $-\mathrm{V}-\mathrm{V} \quad \mathrm{V} \mathbf{V}$

Fig. 10 One rays (top) goes straight through source while the other twists by $\pm \pi$; anagram (bottom)

The probability of Alice seeing a photon at polarizer angle $\theta_{1}$ and Bob simultaneously seeing the other photon at angle $\theta_{2}$ is:

Eigenstate
$\mathrm{A}+\cos \theta_{1} \cos \theta_{1}$
$\cos \theta_{2} \cos \theta_{2}$
B $+\cos \theta_{1}\left(-\sin \theta_{1}\right)$
$\cos \theta_{2} \sin \theta_{2}$
$\mathrm{C}+\left(-\sin \theta_{1}\right) \cos \theta$
$\sin \theta_{2} \cos \theta_{2}$
$\mathrm{D}+\left(-\sin \theta_{1}\right)\left(-\sin \theta_{1}\right) \sin \theta_{2} \sin \theta_{2}$ 


$$
\begin{aligned}
& =\quad \cos \theta_{1} \cos \theta_{1} \quad \cos \theta_{2} \cos \theta_{2} \\
& \breve{\mathrm{G}} \cos \theta_{1} \sin \theta_{1} \quad \cos \theta_{2} \sin \theta_{2} \\
& -\sin \theta_{1} \cos \theta_{1} \quad \sin \theta_{2} \cos \theta_{2} \\
& +\sin \theta_{1} \sin \theta_{1} \quad \sin \theta_{2} \sin \theta_{2}
\end{aligned}
$$

which can be factored:

$$
\begin{aligned}
= & \left(\cos \theta_{1} \cos \theta_{2} \quad \breve{G} \sin \theta_{1} \sin \theta_{2}\right) X \\
& \left(\cos \theta_{1} \cos \theta_{2} \quad \breve{G} \sin \theta_{1} \sin \theta_{2}\right)
\end{aligned}
$$

and simplified:

$$
\begin{gathered}
=\cos \left(\theta_{1}+\theta_{2}\right) \times \cos \left(\theta_{1}+\theta_{2}\right) \\
=\cos ^{2}\left(\theta_{1}+\theta_{2}\right)
\end{gathered}
$$

Our trig shows that the probability of Alice seeing a photon at polarizer angle $\theta_{1}$ and Bob simultaneously seeing the other photon at angle $\theta_{2}$ is $\cos ^{2}\left(\theta_{1}+\theta_{2}\right)$. Table 1 of the Kwiat article (page 5339) says that is precisely what QM predicts and what their experiment found.

\subsection{Translating TEW into QM}

We want to be able to look inside a quantum computer, observe how the bi-rays are arranged, and deduce directly from that, what Bell state is represented. Figures 11 and 12 demonstrate how this can be done. In Figure 11 we will copy Figure 4, but we changed the labels. For example, instead of the polarization of each prong of a bi-ray being $\mathbf{H}$ and $\mathbf{V}$, we are relabeling them as $|+\rangle$ and $|-\rangle$.

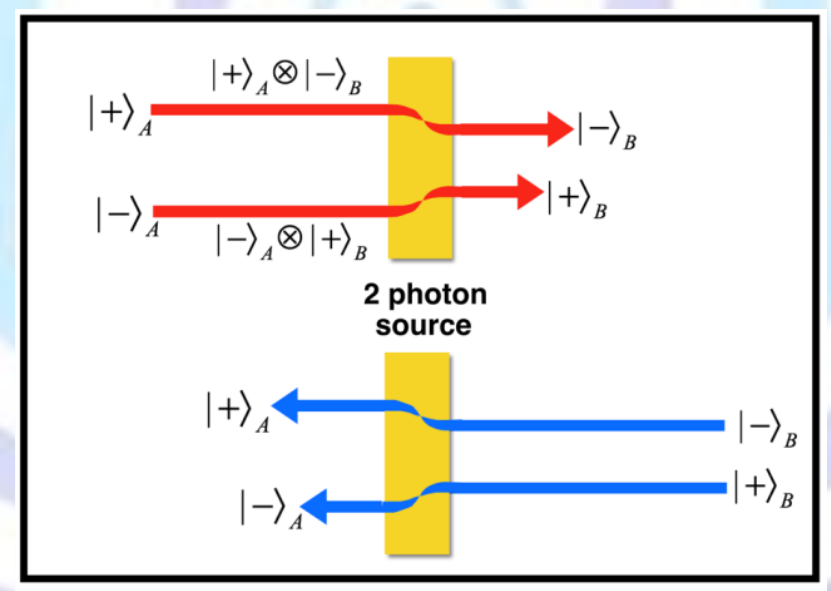

Fig 11. This is Figure 4 except that the labels have been changed

When we read the labels above the red rays in the top half of Figure 11, we see that this represents the first Bell state:

$$
\left(|+\rangle^{\mathrm{H}} \otimes|-\rangle^{\mathrm{B}}\right)-\left(|-\rangle^{\mathrm{U}} \otimes|+\rangle^{\mathrm{B}}\right)
$$

Now we will apply that same technology to Figure 10. Figure 12 is coped from Figure 10, but the labels have been changed. 


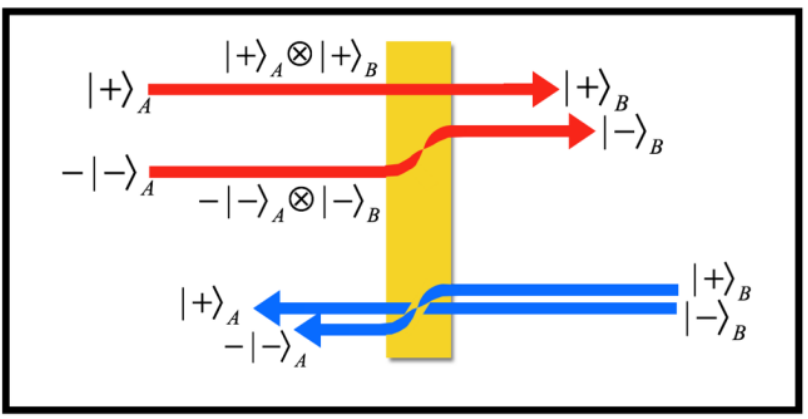

Fig 12. This is from Figure 10 except that the labels have been changed

When we read the labels in Figure 12, we see that this represents the fourth Bell state:

$$
\left(|+\rangle^{Y} \otimes|+\rangle^{B}\right)-\left(|-\rangle^{Y} \otimes|-\rangle^{B}\right)
$$

All we need now is to multiply these equations by the constant $\frac{1}{\sqrt{2}}$ in order to obtain the Bell states $\left|\psi^{+}\right\rangle$and $\left|\xi^{-}\right\rangle$as originally defined:

$$
\begin{aligned}
& \left|\psi^{+}\right\rangle=\frac{1}{\sqrt{2}}\left[\left(|+\rangle_{A} \otimes|-\rangle_{B}\right)+\left(|-\rangle_{A} \otimes|+\rangle_{B}\right)\right] \\
& \left|\xi^{-}\right\rangle=\frac{1}{\sqrt{2}}\left[\left(|+\rangle_{A} \otimes|+\rangle_{B}\right)-\left(|-\rangle_{A} \otimes|-\rangle_{B}\right)\right]
\end{aligned}
$$

Thus we have come full circle.

\subsection{Summary of TEW vis-à-vis the four Bell states}

The four Bell states represent four different bi-rays. The Kwiat article describes a device that can produce all four of the Bell states after polarization is modified using quarter and half plates (not shown in Figure 3). We used the equations from Kwiat's article as a blueprint of how bi-rays work in this equipment.

From that information we reproduced the four EPR-Bell states (Figure 13), as follows:

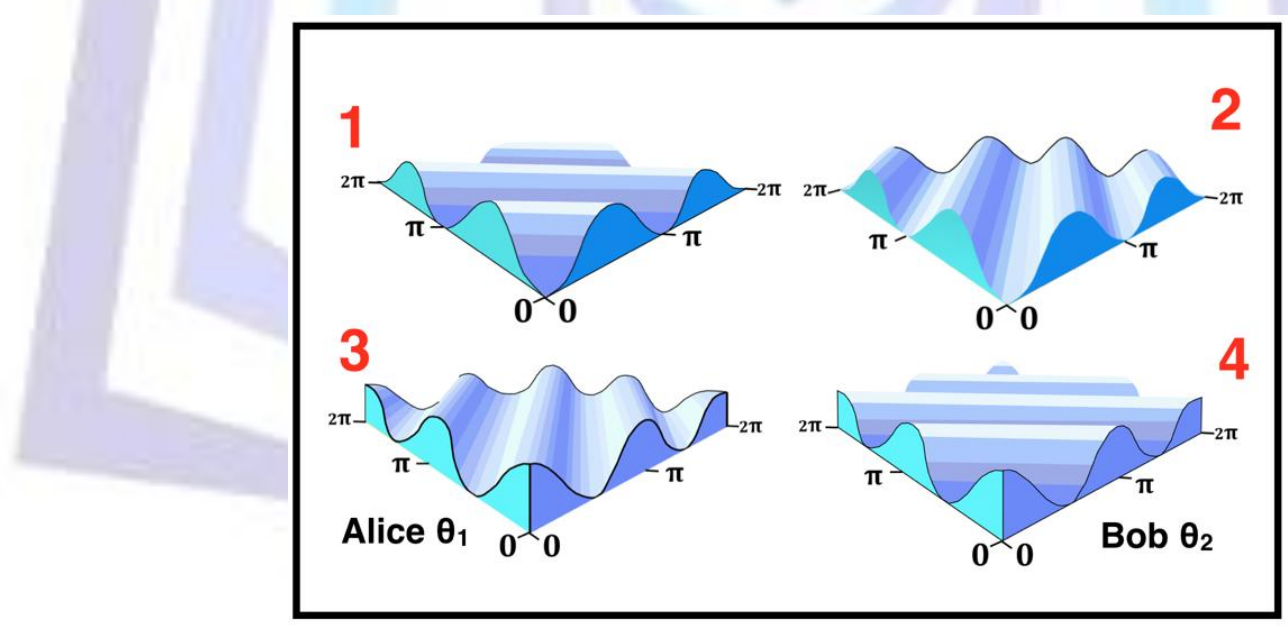

Fig 13. All four Bell states (see red numbers), with coincidence rate $\mathrm{C}$ plotted vertically

In each of these four graphs the horizontal axis on the left represents Alice's polarizer angle $\theta_{1}$, the axis on the right is Bob's polarizer angle $\theta_{2}$, and the vertical axis is the coincidence rate.

The four Bell states present drastically different pictures in Figure 13. In two of them (1 and 4) the waves are north \& south, whereas the waves go in an east-west direction in the other two ( 2 and 3 ). The front corner of each graph takes a value of zero in states 1 and 2 (sine squared at the origin), whereas the front corner takes a value of one in states 3 and 4 (cosine squared at the origin). This startling contrast of the four states is a key to understanding why they are so useful. A noisy information channel might be unable to distinguish two parts of a signal that uses the same one Bell state, but a channel should be able to easily discriminate between different Bell states.

The Kwiat article contains four equations, one for each state. Using those equations as our blueprint, we constructed a diagram of elementary bi-rays in a simple and obvious way. Those diagrams then led to anagrams, which led to trigonometry, the results of which were identical to the results expected from QM, and produced in the Kwiat experiments. Thus we have shown that TEW can account for all four of these states. 
Orthodox quantum information theory asserts that these states cannot be accounted for by local realism. However that referred to the Einstein type of local realism. TEW is a local and realistic model of nature, which IS able to reproduce all four states, as we showed.

\section{PART 5. Explaining entanglement of photons with no shared history}

Entanglement was originally considered a consequence of particles interacting in the past. However, it is now known that particles that share no common history can be entangled.[29-30] A gedanken experiment by Żukowski, Zeilinger, Horne, et.al. uses entanglement swapping and event-ready detectors to generate entangled remote photons (Figure 14). Such techniques were subsequently used by others to build components of quantum computers, such as entanglement between trapped ions, or electrons embedded in NV cavities in distant diamonds .[31-34] The entanglement is confirmed by entanglement swapping with the kind of photons that we are about to discuss. We will review the Żukowski experiment

The top half of Figure 14 is copied from a diagram in Żukowski et al's article. Each of two parametric down converters (PDC) generates a stream of entangled photons, such as the blue and black arrows in Figure 14. Each idler photon (a black arrow) can follow paths c' or $\mathrm{c}$ on the left, and b' or b on the right. Detectors $\mathbf{i}_{1}$ and $\mathbf{i}_{2}$ click when an idler photon arrives. Sooner or later the detectors will spontaneously click simultaneously, which indicates the simultaneous arrival of idler photons from both PDC sources. The window of nanoseconds that defines the word "simultaneous" is narrowed so it is less than the duration of coherence. Under these circumstances two idler photons are entangled (meaning they violate Bell's Theorem). This means the signal photons (blue arrows) are also entangled between the right and left, even though they share no history together. This is called "entanglement swapping" which leads to event-ready detectors.

As stated previously, "entanglement" has different definitions in QM versus TEW. In QM it means two particles share one wave function, so that wave-function collapse is identical for both particles. In TEW it means that particles are following the same bi-ray.

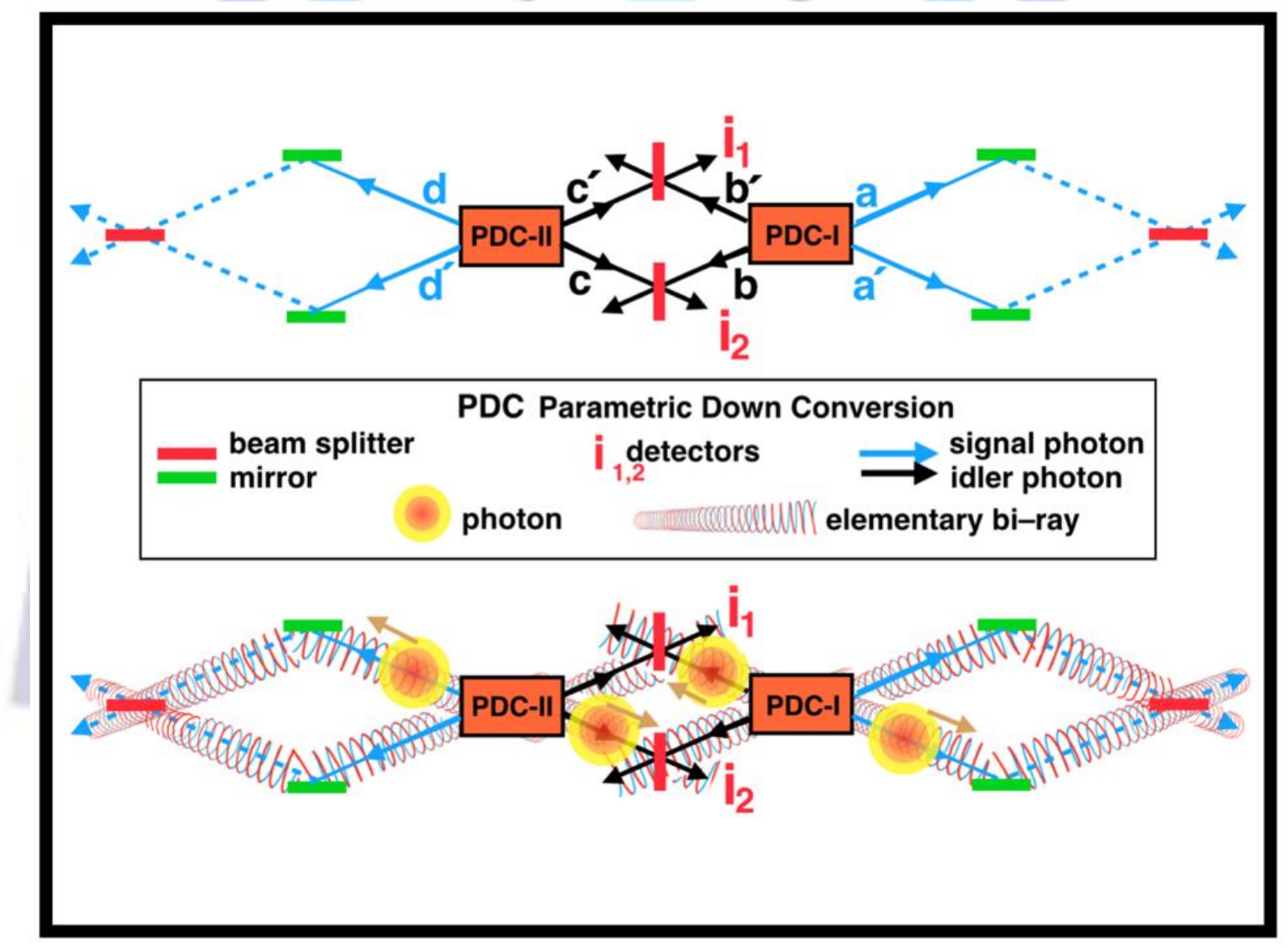

Fig 14. Comparing Żukowski's experiment (top) to the TEW bi-ray explanation (bottom)

The bottom half of Figure 14 shows how TEW explains these phenomena. A helical bi-ray snakes throughout the apparatus: the same one bi-ray has different branches. Four photons (yellow-orange circles) follow this bi-ray. The photons are moving away from the PDC that gave birth to them. Since the helices are all one single bi-ray, therefore all 4 photons are entangled. At the red vertical bars at the center of the apparatus the bi-rays from the left and right intersect at beam splitters. This means that each bi-ray bifurcates at the beam-splitter, so that $1 / 2$ of it is continuous on both sides of the diagram, and the other $1 / 2$ shoots off toward a detector at the periphery. Therefore an idler photon might register at one detector or the other, depending on which direction it takes at the beam splitter.

\section{PART 7. Conclusions}




\subsection{Limitations}

What we accomplished in this article is to sketch an imaginative idea, leaving as many questions and problems unaddressed as does QM. This is how new territory is surveyed on an unexplored continent. Meriwether Lewis and William Clark had to meander through the rivers of the Louisiana territory and make sketchy maps before others could make better maps, inhabit the area, and build railroads and highways.

Our article hinges on images of how elementary bi-rays rotate as they pass through a 2-photon source (for example, Figure 4). That is implied by our starting assumptions about how both entangled photons follow the same bi-ray. There is a peculiarity in the trajectory of such a bi-ray. Figure 2 shows it traveling in a straight line. But in Kwiat's optical equipment it appears to make a sharp turn of at least $165^{\circ}$ inside the BBO crystal. Why? The argon laser sends one photon of wavelength $351.1 \mathrm{~nm}$ toward the BBO crystal. That photon is split at the surface of the crystal into a signal and idler photon of wavelength $702.2 \mathrm{~nm}$, both traveling in the same direction. Those two photons are deflected in different directions in the center of the crystal (along the diagonal where two triangular crystals are glued together). Let's assume that those new photons shoot off with an angle of $15^{\circ}$ between them. The geometry of the elementary ray would involve the bi-ray making a $165^{\circ}$ turn, since it provides a common pathway for both $702.1 \mathrm{~nm}$ photons. While that is an odd idea, there is no reason to reject that idea as we develop a new science of bi-rays. Perhaps bi-rays do such weird things.

\subsection{Two ways of thinking about the metaphysics of mathematics}

QM is based on a "Black Box" theory of nature: we observe what enters an experiment and what comes out, but are advised not to speculate about what happens inside the Black Box. The orthodox QM viewpoint is that our math is not a picture of how nature works independent of the observer. It is a picture of how our meter readings at points input and output are linked.

One of Einstein's many discomforts with QM is that he thought the components of an equation should be analogous to components of physical reality. With relativity that was true. With QM it was not true. But Einstein had an incorrect idea of what constitutes "physical reality" at the quantum scale. If we adopt TEW, the situation changes.

TEW suggests that the guts and internal structure of quantum math $\left|\psi^{+}\right\rangle=\left(\left|H_{1}, V_{2}\right\rangle+\left|V_{1}, H_{2}\right\rangle\right) / \sqrt{2}$ correspond to the guts and internal structure of the real physical world. That structure works in an orderly and predictable way to produce the result $C=\sin ^{2}\left(\theta_{1}+\theta_{2}\right)$. With TEW the human mind can understand how and why nature behaves as she does.

If we can name the ghosts in our world, they are more manageable than if we don't know their names. That ghost who had previously no name, and was called "nonlocality," is now named as "elementary bi-rays." The term "nonlocal" is vague and does not inspire future experiments to investigate how or why it works. If we change the name to "elementary bi-rays" that is a specific term that will inspire future experiments to investigate and control it.

\subsection{TEW explains the Bell test experiments as accurately as QM does}

In this article the four Bell test experiments that can be explained by QM can equally well be explained by TEW. Both theories violate the bound $|S| \leq 2$. According to quantum orthodoxy this means that TEW should be classified as a "nonlocal theory," yet we think of TEW as a local realistic theory. It is "nonlocal" in the sense that the same bi-ray exerts the same influence at both Alice and Bob's equipment. It is local in the sense that a train of ocean waves has palpable local effects against your legs when you stand in the surf.

Repeatedly we have proved that TEW comes up with the same results as QM, and that both theories are equally incompatible with Einstein's version of local realism. Nevertheless many readers do not believe their eyes, cannot accept what they have just seen, and insist that somehow TEW has made the same mistake as Einstein made.

\subsection{Are we making the same mistake as Einstein?}

How does our model differ from Einstein's model? Einstein thought of a hidden variable hidden inside the particle. Our model shows that the mysterious influence is hidden outside the particle, but inside elementary bi-rays in the environment. The bi-rays stretched between Alice and Bob for hours or days before the experiment started.

The nature of these bi-rays is that they carry contingency variables. Given that Alice has chosen polarizer angle $\theta_{1}$, what is the probability of Bob seeing a photon if he sets his polarizer angle at $\theta_{2}$ ? The nature of such a contingency is that it requires information from both Alice and Bob in order to nail down the specifics. This is graphed for the first Bell state in Figure 15. 


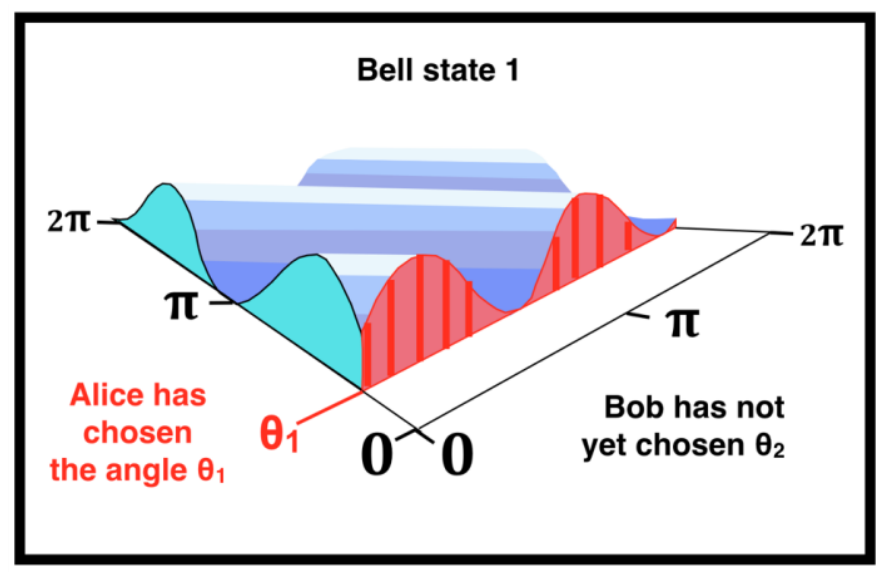

Fig 15. If Alice has chosen $\theta 1$, the probability of Bob seeing a photon at angle $\theta 2$ is the height in red.

According to TEW wavefunction collapse is located inside the 2-photon source. It is not located at the detectors. That "collapse" in TEW means that the photons chose to follow one particular bi-ray among all the competing bi-rays. In Figure 15 the photons chose a bi-ray in Bell state 1 rather than a bi-ray in state 2 or 3 or 4 . By making this choice at birth, the photons have joined a bi-ray that carries those contingency variables.

What does it mean when we say that TEW is a local, realistic theory? First it means that cause and effect are local: a particle on the far side of the galaxy does not instanteously affect a particle here. The causal chain (or wave) is unbroken between Alice and Bob. The "causal chain" is however a chain of contingencies, not a deterministic chain. Finally, it means that the concept of "nonlocality" has been replaced by the concept of an elementary bi-ray.

\subsection{Field theory in TEW}

For 150 years after Newton published Principia Mathematica, most scientists rejected the idea that gravity acted through some kind of medium. They endorsed the idea of direct action at a distance. Newton wrote a letter to Richard Bentley in which he rejected the idea of direct action at a distance, but that view of Newton's was unknown to most scientists. Scientists rejected Faraday's idea of a field through which electromagnetic forces acted. With Maxwell's equations scientific opinion changed and fields were accepted as an explanation of how gravity from the sun affects the planets.[35]

Quantum physics is like the scientists between the time of Newton and Faraday. They reject any sort of intervening medium, and insist on action at a distance. TEW says there is an intervening medium, namely elementary rays. However, when we look at TEW as a field theory we find something peculiar, namely the absence of any variable specifying physical location. Gravity diminishes with the square of the distance, but the equations considered in this article contain no variable specifying location. Look at the first Bell equation:

$$
\left|\psi^{+}\right\rangle \equiv \frac{1}{\sqrt{2}}\left[\left(|+\rangle_{A} \otimes|-\rangle_{B}\right)+\left(|-\rangle_{A} \otimes|+\rangle_{B}\right)\right]
$$

There is no variable in that equation for physical location! Whether you are located in Alice's neighborhood, or in the vicinity of the source, or near Bob, the equation remains unchanged. The same is true of the two equivalent formulas we derived:

$$
\begin{aligned}
\left|\psi^{+}\right\rangle & =\frac{1}{\sqrt{2}}\left(\left|H_{A}, \mathrm{~V}_{B}\right\rangle+\left|V_{A}, \mathrm{H}_{B}\right\rangle\right) \\
C & =\sin ^{2}\left(\theta_{1}+\theta_{2}\right)
\end{aligned}
$$

Thus TEW introduces a field theory in which there is a uniform field over distance. No wonder quantum physicists believe that their equations stand outside the space-time continuum! According to TEW quantum equations describe actual physical nature inside the space-time continuum, but the distance variable is totally weird.

If you are always dealing with the same elementary bi-ray, it is uniform over distance. Only when you change from one biray to another does distance matter. If your source shifted from the first Bell state to the fourth Bell state, that change would propagate out at the speed of light. This is important to remember when we talk about quantum communication. If a particle source is spelling out a message by shifting from one Bell state to succession of other Bell states, distance and the speed of light become relevant factors.

\section{References}

[1] J. Baggott, The Quantum Story, Oxford University Press, 2011, see pages 105-111.

[2] J. H. Boyd, "A paradigm shift in mathematical physics, Part 3: A mirror image of Feynman's quantum electrodynamics 
(QED)," Journal of Advances in Mathematics 11 (\#2) (August, 2015).

[3] J. H. Boyd, "A paradigm shift in mathematical physics, Part 2: A new local realism explains Bell test \& other experiments," Journal of Advances in Mathematics, 10, \#9, (July 2015).

[4] J. H. Boyd, "A paradigm shift in mathematical physics, Part 1: The Theory of Elementary Waves (TEW)," Journal of Advances in Mathematics 10, (\#9) (June 2015).

[5] J. H. Boyd, A new variety of local realism explains a Bell test experiment: the Theory of Elementary Waves (TEW) with no hidden variables, Journal of Advances in Physics 8, 2051-58 (Mar 2015).

[6] J. H. Boyd, The Theory of Elementary Waves eliminates Wave Particle Duality, Journal of Advances in Physics 7, 1916-1922 (Feb 2015).

[7] J. H. Boyd, Re-thinking Alain Aspect's 1982 Bell test experiment with delayed choice, Physics Essays 26 (4) $582-591$ (2013).

[8] J. H. Boyd, Re-thinking a delayed choice quantum eraser experiment: a simple baseball model, Physics Essays 26, 100-109 (March 2013), (doi: 10.4006/0836-1398-26.1.100).

[9] J. H. Boyd, Rethinking a Wheeler delayed choice gedanken experiment, Physics Essays 25, (3) pp. 390-396, 2012. doi: http://dx.doi.org/10.4006/0836-1398-25.3.390

[10] L. E. Little, Theory of Elementary Waves, Physics Essays 9 (1), 100-134 (1996).

[11] L. E. Little, Theory of Elementary Waves, (New Classics Library, New York, 2009).

[12] J. S. Bell, “On the Einstein Podolsky Rosen paradox," Physics 1, 195-200 (1964).

[13] J. S. Bell, "The paradox of Einstein, Podolsky and Rosen: action at a distance in quantum mechanics?," Speculations in Science and Technology, 10, no. 4, 269-285 (1987).

[14] J. S. Bell, "Bertlmann's socks and the nature of reality," Journal de Physique, 42, Colloque C2, supplément au No3, S. 41 (1981).

[15] M. A. Nielsen and I. Chuang, Computation \& Quantum Information, (Cambridge U. Press, 2010) from

https://books.google.com/books?hl=en\&lr=\&id=-s4DEy7o-

a0C\&oi=fnd\&pg=PR17\&dq=quantum+information+science\&ots=NG5HcrpBZx\&sig=s1JK2ZsPVSdJz5kshj8xQYhq_gY\#v=

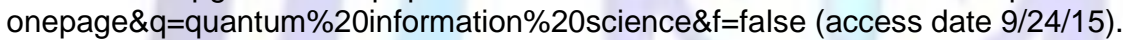

[16] S. Bone and M. Castro, "A brief history of quantum computing," from the Department of Computing, Imperial College of London (the document has no date stated). Available online at http://www.doc.ic.ac.uk/ nd/surprise_97/journal/vol4/spb3/(access date Oct 1, 2015).

[17] (No author and no date listed) "Quantum computing," from a Canadian website: "A review of the universe - structures, evolutions, observations, and theories." This series of four linked websites on quantum computing are online at https://universe-review.ca/R13-11-QuantumComputing.htm and https://universe-review.ca/R13-11QuantumComputing01.htm and https://universe-review.ca/R13-11-QuantumComputing02.htm https://universereview.ca/R13-11-QuantumComputing03.htm (accessed 10/2/2015).

[18] P. Hemmer and J. Wrachtrup, "Where is my quantum computer?" Science 324, 473-474 (April 24, 2009). DOI: 10.1126/science.1170912

[19] P. G. Kwiat, K. Mattle, H. Weinfurter, A. Zeilinger, A.V. Serbienko and Y. Shih, "New high-intensity source of polarization-entangled photon pairs," Physical Review Letters, 75 (\#24) pp. 4337-4341 (1995).

[20] A. Einstein, B. Podolsky, and N. Rosen, "Can quantum-mechanical description of physical reality be considered complete?" Physical Review, 47, 777-780 (1935).

[21] N. D. Mermin, The Journal of Philosophy, 78, \# 7, 397-408 (July, 1981).

[22] N. D. Mermin, "Is the moon there when nobody looks? Reality and the quantum theory," Physics Today, 38, 38-47 (April 1985).

[23] J. F. Clauser, M.A. Horne, A. Shimony and R. A. Holt (CHSH), "Proposed experiment to test local hidden-variable theories," Physical Review Letters, 23 (\#15), pp. 880-884 (1969).

[24] A. Aspect, J. Dalibard, G. Roger, "Experimental test of Bell's inequalities using time-varying analyzers," Physical Review Letters, 49, 1804-1807 (1982).

[25] B. S. Cirel'son, "Quantum generalizations of Bell's inequality," Letters in Mathematical Physics, 4, 93-100 (1980) available online at http://link.springer.com/article/10.1007/BF00417500\#page-1 , (access date June 5, 2015).

[26] R. P. Feynman, QED (Oxford University Press and Princeton U. Press, 1985).

[27] [No author listed], "Bell States, Bell Inequalities," 8/28/2003, Lecture 2.PDF from Chem/CS/Phys191: Qubits, Quantum Mechanics, and Computers, EECS Instructional and Electronics Support, University of California, Berkley. (available at http://www-inst.eecs.berkeley.edu/ cs191/sp05/lectures/lecture2.pdf. Access date Oct 11, 1015). 
[28] A. Dousse, J. Suffczyński, A. Beveratos, O. Krebs, A. Lemaître, I. Sagnes, J. Bloch, P. Voisin \& P. Senellart, "Ultrabright source of entangled photon pairs," Nature 466, 217-220 (08 July 2010) doi:10.1038/nature09148

[29] B. Yurke and B. Stoler, Einstein-Podolsky-Rosen effects from independent particle sources, Physical Review Letters 68, 1251-1254 (1992).

[30] M. Żukowski, Zeilinger, A., Horne, M. A. \& Ekert, A. K. "Event--ready--detectors" Bell experiment via entanglement swapping. Physical Review Letters 71, 4287-4290 (1993).

[31] S. D. Barrett and P. Kok, Efficient high -fidelity quantum computation using matter qubits and linear optics. Physical Review A 71, 060310 (2005).

[32] H. Bernien, B. Hensen, W. Pfaff, G. Koolstra, M. S. Blok, L. Robledo, T. H. Taminiau, M. Markham, D. J. Twitchen, L. Childress \& R. Hanson, Heralded entanglement between solid-state quits separated by three meters. Nature 497, 86-90 (2013).

[33] J. Hofmann, M. Krug, N. Ortegel, L. Gérard, M. Weber, W. Rosenfeld, H. Weinfurter, "Heralded entanglement between widely separated atoms," Science 337, 72-75 (6 July 2012)

[34] B. Hensen, H. Bernien, A. E. Dréau, A. Reiserer, N. Kalb, M. S. Blok, et.al., "Loophole-free Bell inequality violation using electron spins separated by 1.3 kilometres," Nature 526, (2015), doi: 10.1038/nature15759.

[35] B. Mahon, The man who changed everything: the life of James Clerk Maxwell (Wiley Publishing, Hoboken, New Jersey, 2004), chapter 5.

\section{Author's biography with Photo}

Jeffrey H. Boyd

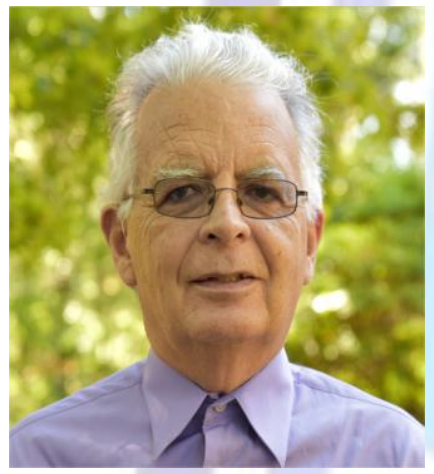

Dr. Boyd was born in 1943 in northern New Jersey, USA, the son of a factory worker family in which no one had ever been to college. In high school he helped his father dig a basement by hand, using a pick, shovel and wheelbarrow. Boyd chose which college to apply to based on which one had the best applied math department. Boyd's undergraduate degree in mathematics was from Brown University in 1965. He has post-graduate degrees from Harvard, Yale and Case Western Reserve Universities, has served on the research faculty of the National Institutes of Health for seven years, and has been on the faculty of the Yale Medical School. His day job is as a physician: a psychiatrist. Boyd retired after a quarter century at Waterbury Hospital, Waterbury CT, a Yale teaching hospital where he had served as chairman of behavioral health and chairman of ethics. Almost half a century ago Boyd abandoned his first love (mathematics) because of his belief that no mathematician over the age of 25 ever discovered anything important, and he was rapidly approaching that age. He wanted to be in a field where age and experience counted for you, not against you. A rewarding career in medicine followed. Then Andrew Wiles proved Fermat's last theorem at age forty and Lewis Little discovered elementary waves at age fifty-two. With this series of articles in JAM the author discovered, to his astonishment, that age is not the main determinant of whether there are mathematical neurons in one's skull. Boyd has published in the New England Journal of Medicine, Journal of Advances in Physics, Journal of Advances in Mathematics and Physics Essays. 